\title{
Deriving Acceptable Biological Catch from the Overfishing Limit: Implications for Assessment Models
}

\author{
Michael H. Prager* and Kyle W. Shertzer \\ National Oceanic and Atmospheric Administration, National Marine Fisheries Service, Southeast Fisheries \\ Science Center, 101 Pivers Island Road, Beaufort, North Carolina 28516 USA
}

\begin{abstract}
The recently revised Magnuson-Stevens Fishery Conservation and Management Act requires that U.S. fishery management councils avoid overfishing by setting annual catch limits (ACLs) not exceeding recommendations of the councils' scientific advisers. To meet that requirement, the scientific advisers will need to know the overfishing limit (OFL) estimated in each stock assessment, with OFL being the catch available from applying the limit fishing mortality rate to current or projected stock biomass. The advisers then will derive "acceptable biological catch" ( $\mathrm{ABC}$ ) from OFL by reducing OFL to allow for scientific uncertainty, and $\mathrm{ABC}$ becomes their recommendation to the council. We suggest methodology based on simple probability theory by which scientific advisers can compute $\mathrm{ABC}$ from OFL and the statistical distribution of OFL as estimated by a stock assessment. Our method includes approximations to the distribution of OFL if it is not known from the assessment; however, we find it preferable to have the assessment model estimate the distribution of OFL directly. Probability-based methods such as this one provide well-defined approaches to setting ABC and may be helpful to scientific advisers as they translate the new legal requirement into concrete advice.
\end{abstract}

In 2006, the U.S. Congress reauthorized the Magnuson-Stevens Fishery Conservation and Management Act. The reauthorizing statute (MagnusonStevens Reauthorization Act [MSRA]; MSRA 2006) established several new requirements for U.S. federal fishery management. Two widely noted requirements are that fishery management councils, which manage U.S. fisheries in federal waters, must set annual catch limits (ACLs) on managed stocks and that those limits may not exceed the recommendations of a council's scientific advisers.

To translate this law into practice, the National Marine Fisheries Service has developed National Standards Guidelines (U.S. Office of the Federal Register 2009) as revisions to the U.S. Code of Federal Regulations. The guidelines refer to two main classes of uncertainty: (1) scientific uncertainty, which embodies uncertainties in scientific understanding, data, and estimation; and (2) management uncertainty, which

\footnotetext{
* Corresponding author: mike.prager@noaa.gov
}

Received July 16, 2009; accepted September 18, 2009 Published online January 14, 2010 reflects the uncertain realization of management regulations in a fishery. The guidelines suggest that usually the scientific and statistical committee (SSC) of each council will be the scientific adviser and that the SSC's recommendations, which are not to be exceeded, should include the acceptable biological catch (ABC) computed by reducing the overfishing limit (OFL) to allow for scientific uncertainty. In this context, OFL is defined as the catch available from the projected stock biomass $(B)$ at the fishing mortality rate's $(F)$ limit reference point $\left(F_{\text {lim }}\right)$. By default, $F_{\text {lim }}$ equals $F_{\text {MSY }}$, the $F$ at which maximum sustainable yield can be obtained from a stock in equilibrium (U.S. Office of the Federal Register 2009).

Here, we describe a framework that can be used to choose ABC given three things: the OFL, the statistical distribution of OFL, and the allowable probability of overfishing. Ideally, the distribution of OFL will be available from the stock assessment. If not, the distribution can be computed by propagation-of-error methods. We examine, through four examples, how well propagation-of-error methods might perform in this application.

\section{Probability-Based Reference Points}

Several authors have demonstrated uses of probability theory to derive fishery reference points (typically denoted "targets" and "limits") that incorporate various kinds of uncertainty. Caddy and McGarvey (1996) described a procedure to set a target $F$ given a precisely known $F_{\text {lim }}$ so that the rate realized in the next period $\left(F_{\text {next }}\right)$ would exceed $F_{\text {lim }}$ with only some specified probability $P^{*}$ (Figure $1 \mathrm{a}$ ). The procedure assumes that $F_{\text {next }}$ will be centered on the target but will probably not equal it because of imperfect implementation of management controls (e.g., quota overruns) or imperfect stock assessment.

Prager et al. (2003) revised and extended the work of Caddy and McGarvey (1996) in several ways. The revised procedure, which they termed REPAST, allows uncertainty both in estimating the $F_{\text {lim }}$ (a type of scientific uncertainty) and in attaining the target (a type of management uncertainty); uses ratios to reduce possible covariance between quantities; and can be applied to reference points in $B$ as well as $F$. Prager et 

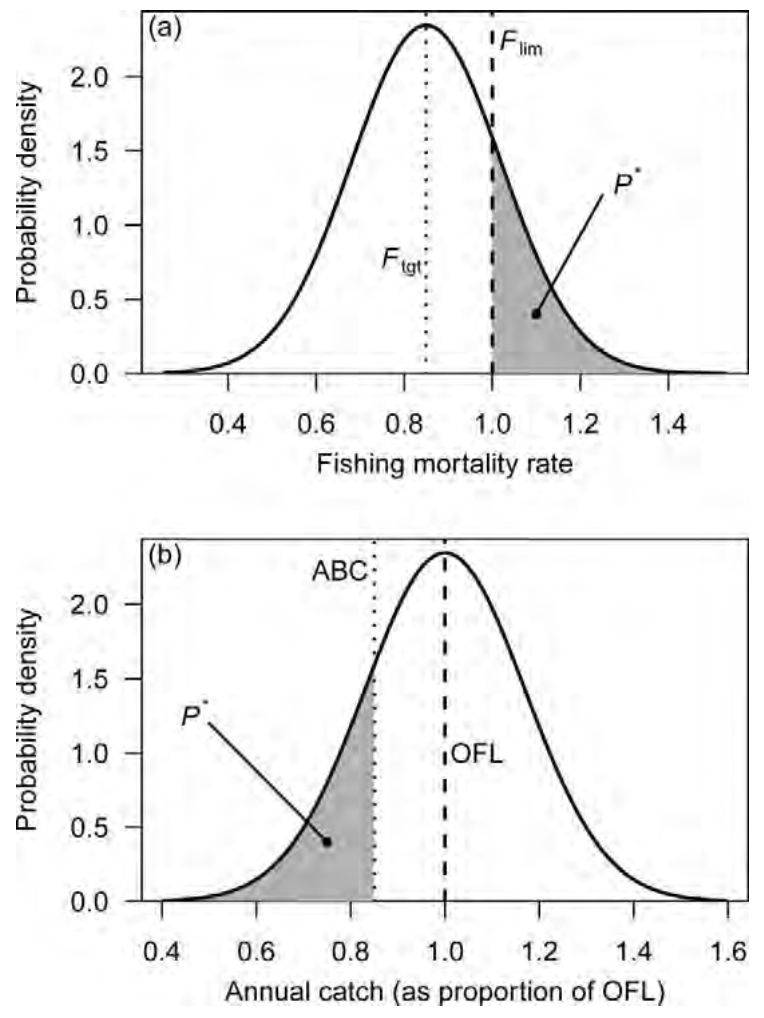

FIGURE 1.-(a) Method of Caddy and McGarvey (1996) for deriving a target reference point from a limit reference point. Given the distribution of the realized fishing mortality rate $(F)$ around its target $\left(F_{\text {tgt }}\right)$, the limit reference point $\left(F_{\text {lim }}\right)$ is adjusted so that the probability (shaded area) that realized $F$ exceeds $F_{\text {lim }}$ equals the preset value $P^{*}$. (b) Proposed procedure for setting acceptable biological catch $(\mathrm{ABC})$ from the statistical distribution of the overfishing limit (OFL) is shown. Given the distribution of OFL, ABC is adjusted so that the probability of $\mathrm{ABC}$ exceeding $\mathrm{OFL}$ is equal to the preset value $P^{*}$.

al. (2003) suggested that an adjustment (bias correction) be made when the distribution of past catches has not been centered on corresponding targets. Such an adjustment would address the problem of "regulatory slippage" noted by Eagle and Thompson (2003).

Shertzer et al. (2008) described a procedure (PASCL) that was extended considerably from that of Prager et al. (2003) and was intended for setting ABCs, ACLs, and annual catch targets (ACTs) in a series of several years, generally the period from one stock assessment until the next. (The relationship among reference points is ACT $\leq \mathrm{ACL} \leq \mathrm{ABC} \leq \mathrm{OFL}$, with at least one of the inequalities being strict; use of an ACT is optional [U.S. Office of the Federal Register 2009].) The Shertzer et al. (2008) procedure uses a stochastic projection model starting from estimates of $F_{\text {lim }}$ and terminal-year abundance; it can incorporate major forms of scientific uncertainty and management uncertainty.

\section{Single-Year Computations}

In this article, we describe a simple probabilistic approach related to those previous methods. Rather than computing multiyear ABCs and ACTs, as in PASCL, this simpler approach computes only a single year's $\mathrm{ABC}$ given a projected $B$ and an estimate of the distribution of OFL under scientific uncertainty. Because that distribution may be unavailable, we examine a group of methods for approximating it from the variances of the projected $B$ and $F_{\text {lim }}$ and possibly from their covariance.

We present this work because we expect that some fishery management councils will take a stepwise approach (i.e., annually) in complying with the new federal requirements. In one step, each council will ask its scientific advisers for annual $\mathrm{ABCs}$ for a managed stock or stocks, derived from the OFL and taking scientific uncertainty into account as required (U.S. Office of the Federal Register 2009). In a later step, each council will set ACLs (and possibly ACTs) from those ABCs, taking management uncertainty into account. This paper addresses only the first step, the work of the scientific advisers. In the language of Eagle and Thompson (2003), methods like REPAST control the probability of both the scientific and regulatory forms of overfishing, whereas the present method controls only scientific overfishing.

We propose setting $\mathrm{ABC}$ from the statistical distribution of OFL, which in many cases can be estimated by stock assessment software that is suitably programmed. If so, one can apply a procedure like that of Caddy and McGarvey (1996) to set ABC less than OFL such that the probability of ABC exceeding OFL (i.e., $P[\mathrm{ABC}>\mathrm{OFL}]$ ) equals some chosen value $P^{*}$ (Figure 1b). In other words, we propose that the ABC be chosen as the percentile of the distribution of OFL that results in a $P\left(\mathrm{ABC}>\mathrm{OFL}\right.$ ) equal to $P^{*}$ (Figure 1b). This is a mirror image of the approach of Caddy and McGarvey (1996) in that they considered the $F_{\text {lim }}$ to be fixed and the corresponding target to be uncertain, whereas here the situation is reversed: the limiting value (OFL) is uncertain and the $A B C$ will be expressed as a point value (Figure 1). This probabilistic approach requires two inputs: the value of $P^{*}$ and the distribution of OFL-that is, its central tendency and some description, either empirical or parametric, of the uncertainty around that central value (Figure 1b).

\section{Approximating the Overfishing Limit Distribution}

Ideally, the distribution of OFL will be estimated by the stock assessment software. However, the distribution is not available from all commonly used software. If the available software estimates the distributions (or 
simply the variances) of $F_{\text {lim }}$ and current or projected $B$, propagation-of-error methods can be used to approximate the distribution of OFL.

To accomplish that, error is propagated through the catch equation (the equation giving catch as a function of stock size, $F$, and other factors) used to model the stock. Letting $F$ equal $F_{\text {lim }}$ and disregarding age structure, the Baranov catch equation, widely used in age-structured fishery models, expresses the OFL as a function $(G)$ of $F_{\lim }$ and $B$ :

$$
\mathrm{OFL}=G\left(F_{\lim }, B\right)=\frac{F_{\lim } B\left(1-e^{-M-F_{\lim }}\right)}{F_{\lim }+M},
$$

where $M$ is the natural mortality rate. If the assessment is based on a different catch equation, such as the logistic stock-production catch equation (Prager 1994), the OFL will still be a function of $F_{\lim }$ and $B$, and possibly other factors.

We considered two methods for examining propagation of error. The delta method, an approximation based on Taylor series (Seber 1973), has been applied in many contexts, including fishery stock assessment models (e.g., Prager and MacCall 1988). Its main advantage here would be computational economy because of the existence of analytical derivatives of the Baranov and logistic catch equations. Its disadvantage would be its requirement to assume a parametric form (usually normal) for the probability distributions of $B, F_{\text {lim }}$, and OFL. The main competing method, Monte Carlo simulation, does not require that assumption. Its drawback, computational intensity, is not significant in this application, given the abilities of today's computers. Thus, we chose Monte Carlo simulation for this study.

Monte Carlo simulation is straightforward to implement through such computer packages as WinBUGS (Lunn et al. 2000) or R (R Development Core Team 2008); it can accommodate any distributions of $F_{\text {lim }}$ and $B$, including empirical distributions; and it estimates a complete distribution of OFL, not just parameters of a given distributional form. How well does Monte Carlo simulation work in this context? It seems unlikely that any simulation study could answer that question exhaustively, given the very many stocks and assessment models of interest. To obtain some preliminary impressions, we examined results of four stock assessments by using two assessment models, and we compared the results of four Monte Carlo configurations with the direct estimates of OFL distributions.

\section{Methods}

Of our four cases, three were taken from recent southeastern U.S. stock assessments of black sea bass Centropristis striata, red porgy Pagrus pagrus, and tilefish Lopholatilus chamaeleonticeps; the fourth case was from analysis of a historical data set describing swordfish Xiphias gladius. Black sea bass, red porgy, and swordfish were analyzed with a nonequilibrium logistic production model (Schaefer 1957; Pella 1967) in the formulation of Prager (1994) as modeled with ASPIC software (Prager 1995). The tilefish data were analyzed with a statistical catch-age model for which form and implementing software were generally similar to those of Methot's (1989) stock synthesis model. The catch-age model and software are described in detail in the assessment report (SEDAR 2004).

Each assessment estimated the distribution of OFL or the distributions of its components ( $B$ and $F_{\text {lim }}$ ), from which we computed the distribution of OFL by application of the corresponding catch equation to paired realizations of $B$ and $F_{\text {lim }}$. The ASPIC software uses bootstrapping to estimate the OFL distribution, which we bias-corrected (Efron 1987) before use. The catch-age model uses a mixed Monte Carlo and bootstrap approach (Legault et al. 2001) to estimate the OFL distribution, and in this case the distribution did not require bias correction. In each case, the OFL distribution from the assessment was used as a reference value.

Some assessment software may estimate means and variances of $F_{\lim }$ and $B$ and possibly the covariance between them but not the empirical OFL distribution itself. For example, models that use the Hessian matrix to derive variances assume that distributions are asymptotically normal. We evaluated four configurations of a Monte Carlo approximation that could be used in such cases. Configuration 1 assumed normality of $F_{\lim }$ and $B$ in arithmetic space with zero covariance between them; configuration 2 assumed normality in log space with zero covariance; configuration 3 assumed normality in arithmetic space with covariance known; and configuration 4 assumed normality in log space with covariance known.

We programmed Monte Carlo simulations in the statistical language $\mathrm{R}$ ( $\mathrm{R}$ Development Core Team 2008) using the function "rnorm" to generate univariate normal random numbers and the function "mvrnorm" from package MASS (Venables and Ripley 2002) to generate bivariate normal random numbers with nonzero covariance. In each simulation, 1,000 draws were made from the univariate distributions of $F_{\lim }$ and $B$ or from the bivariate distribution of $F_{\text {lim }}$ and $B$. Each draw was transformed by the corresponding catch equation (equation 1 in the agestructured example; equation 6 of Prager [1994] in the production model examples) into a corresponding value of OFL. The resulting 1,000 values of OFL were taken together to approximate the distribution of 

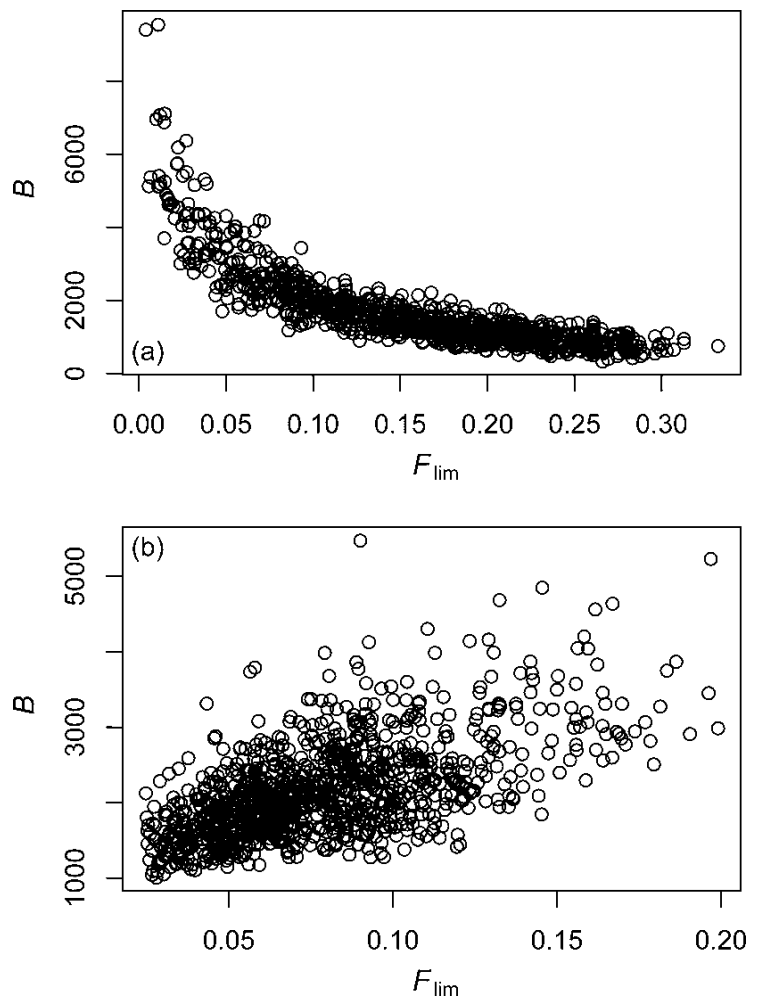

Figure 2.-Bivariate distribution of estimates of the limit fishing mortality rate $\left(F_{\text {lim }}\right)$ and biomass $(B)$ in the next year from (a) a nonequilibrium production model of black sea bass and (b) a statistical catch-age model of tilefish.

OFL. As when the distribution was known directly from the stock assessment, $\mathrm{ABC}$ was taken as the percentile of the OFL distribution corresponding to $P^{*}$.

For each stock, the $\mathrm{ABC}$ was computed from the reference OFL distribution and from each Monte Carlo configuration and was evaluated with values of $P^{*}$ from 0.2 to 0.5 in steps of 0.05 . We examined graphically the consequences to $\mathrm{ABC}$ that would ensue from using each of the four approximations in place of corresponding percentiles of the reference distribution of OFL. We also tabulated relative differences from the reference $\mathrm{ABCs}$ at a $P^{*}$ of 0.35 , which is a midrange, representative value.

\section{Results}

Correlations between estimates of $F_{\text {lim }}$ (here, $F_{\text {lim }}=$ $F_{\text {MSY }}$ ) and $B$ varied by the type of assessment model from which they were obtained. Estimates from the three production models were negatively correlated; results for black sea bass (Figure 2a) are typical. In contrast, estimates from the statistical catch-age model used for tilefish were positively correlated (Figure 2b). In all four cases, the correlation was significantly different from zero $(P<0.0001)$.
When $\mathrm{ABC}$ was approximated with the Monte Carlo configurations, the differences from the reference $\mathrm{ABC}$ were considerably larger in analyses of black sea bass and swordfish (Figures 3a, b) than in analyses of red porgy and tilefish (Figures 3c, d). Most large differences were negative; that is, use of the approximations gave lower estimates of ABC (Table 1). The Monte Carlo configuration that most closely approached the reference $A B C$ varied by stock, but when we considered only the two stocks (black sea bass and swordfish) that exhibited large differences in performance among configurations, the lognormal configuration with known covariance performed appreciably better than other Monte Carlo configurations (Table 1).

\section{Discussion}

Our results imply that when using probability-based methods, it is highly desirable for the assessment model to generate an estimated distribution of OFL because estimates of $\mathrm{ABC}$ derived from our approximations could differ from the reference values considerably (Figure 3). In the two more extreme cases considered (black sea bass and swordfish), the approximations of $\mathrm{ABC}$ were too low, although we have no reason to believe that this is a general result. If an estimate of the OFL distribution is not available, Monte Carlo simulation can be used to approximate it as long as the assessment provides estimates of the components $\left(F_{\lim }\right.$ and $B$ ) of OFL and of their variances. In this application, Monte Carlo simulation tended to perform better when the covariance between $F_{\lim }$ and $B$ was also available. Our results (Figure 3; Table 1) suggest that among the Monte Carlo configurations examined, the one in logarithmic space with covariance (i.e., configuration 4) performed best and therefore should be used when possible. Unfortunately, we can make no ad hoc recommendation about a value that might be assumed for covariance when an estimate is not available, because in our examples the sign of the covariance depended on the assessment model used (Figure 2).

The production model used here (Prager 1994, 1995) incorporates observation error (e.g., error in the abundance index) but not process error (e.g., variability in production at a given $B$ ), and like most production models, this model has few estimated parameters. The relative inflexibility of that modeling scheme almost certainly contributes to the consistent pattern of negative correlation seen between estimates of terminal $B$ and $F_{\text {lim }}$ (Figure 2a). The age-structured model, in contrast, incorporates process error in recruitment as well as observation error in the data and has dozens of estimated parameters. We have found that its correlation patterns vary among applications. The positive relationship of Figure $2 \mathrm{~b}$ appears to be driven by 

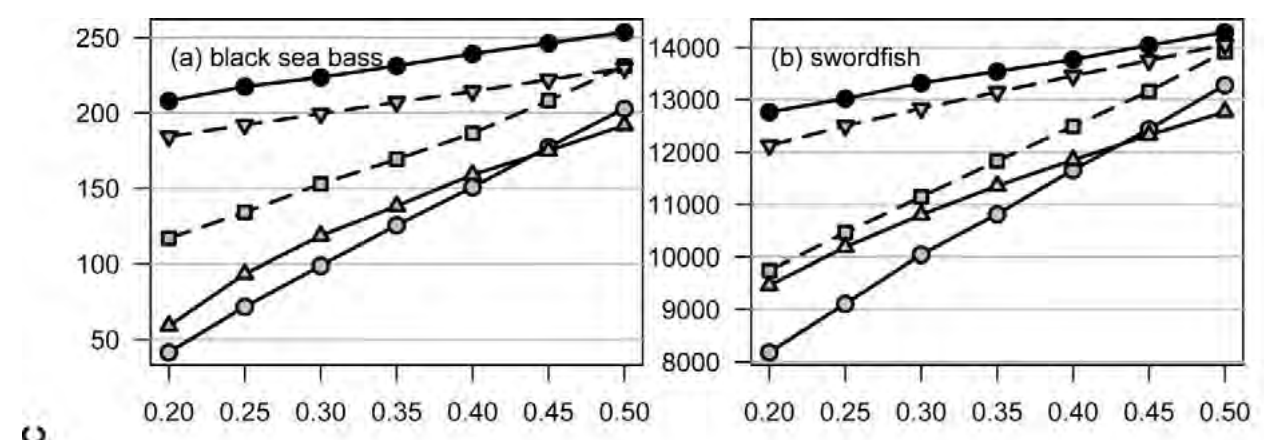

$\stackrel{0}{4}$
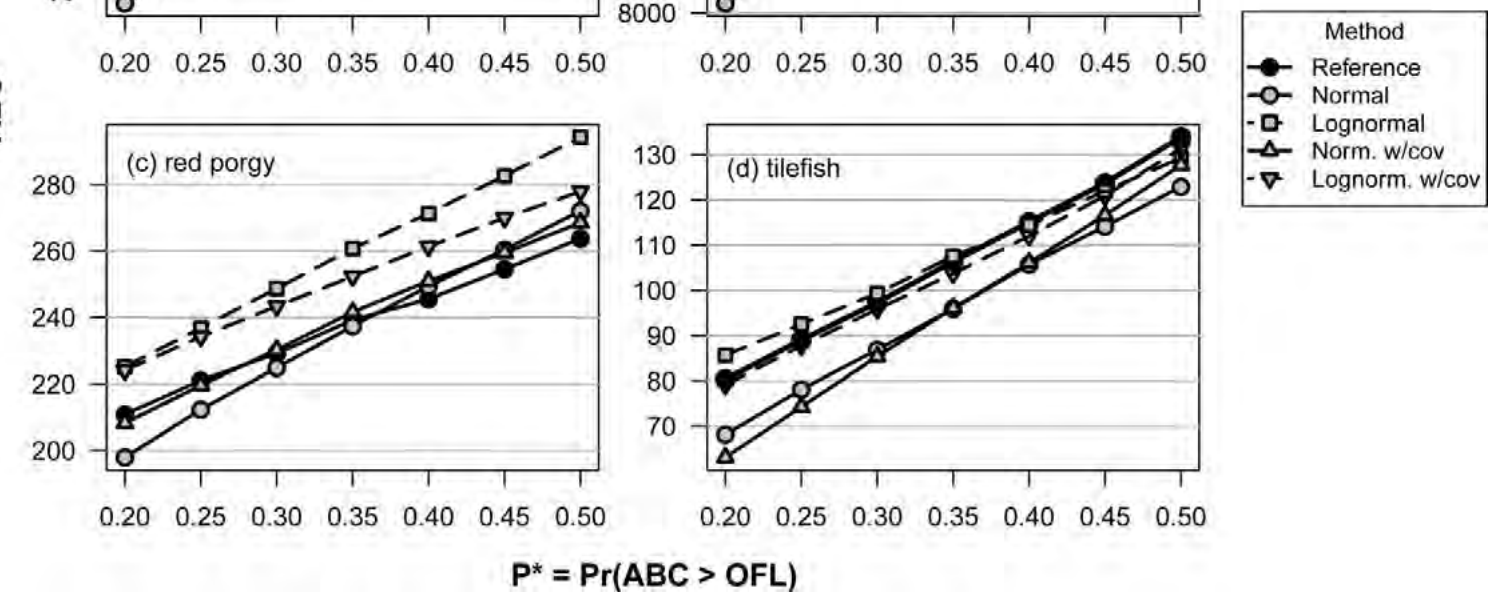

FiguRE 3.-Comparison of acceptable biological catch (ABC) determinations over a range of $P^{*}$, where $P^{*}$ is the allowable probability that $\mathrm{ABC}$ will exceed the overfishing limit (OFL). Reference $\mathrm{ABCs}$ were determined based on OFL distributions from stock assessments; other ABCs were computed from four Monte Carlo configurations that approximated the OFL distribution (see Methods for details; norm = normal distribution; lognorm = lognormal distribution; $\operatorname{cov}=$ covariance). Examples are shown for (a) black sea bass, (b) swordfish, (c) red porgy, and (d) tilefish.

variation in $M$, which correlates positively with both terminal $B$ and $F_{\text {lim. }}$.

Several caveats should be kept in mind if applying the methods described here. Because statistical assumptions are rarely (if ever) met in fisheries analyses, picking a percentile from the distribution of OFL is unlikely to result in a perfectly realized probability of overfishing. One issue is that small values of $P^{*}$ (say, $P^{*}<0.2$ ) may produce inconsistent results because distributions that vary from their estimates tend to do so most strongly in the tails. Another issue is that if precision estimates from an assessment are unreliable or if the assessment has not exhibited stability over time, it may be desirable to explore other methods, such as ad hoc proportional reductions of catch from OFL or of $F$ from $F_{\text {lim }}$. An approach being explored by the South Atlantic Fishery Management Council and the Mid-Atlantic Fishery Management Council is to use a framework similar to this one but to reduce $P^{*}$ from a baseline value in response to various factors. These factors might include excessive time since the last assessment, known data limitations, incomplete variance estimation, status of the stock, or life history characteristics that increase vulnerability or susceptibility.

What sort of values are appropriate for $P^{*}$, either for direct application or to be adjusted as just described?

TABLE 1.-Percentage differences in acceptable biological catch (ABC) between values directly estimated by an assessment model (reference values) and those obtained by using four configurations of Monte Carlo simulation on abbreviated assessment estimates. A statistical catch-age model was used for tilefish; a nonequilibrium production model was used for the other species. All values were computed using a $P^{*}$ of 0.35 , where $P^{*}$ is the allowable probability that $\mathrm{ABC}$ exceeds the overfishing limit.

\begin{tabular}{lcccc}
\hline & \multicolumn{3}{c}{ Species } \\
\cline { 2 - 5 } Monte Carlo approximation configuration & Black sea bass & Swordfish & Red porgy & Tilefish \\
\hline Normal & -48.3 & -21.6 & -0.6 & -11.0 \\
Lognormal & -24.9 & -12.3 & 9.6 & 0.6 \\
Normal with covariance & -39.8 & -16.4 & 0.6 & -8.2 \\
Lognormal with covariance & -10.3 & -2.6 & 5.5 & -1.6 \\
\hline
\end{tabular}


Because that is a policy issue and also may vary by application, no definitive or universal answer can be given. As noted earlier, setting ABC less than OFL is an early step in a longer process. A council is then charged with setting an ACL (and possibly an ACT) for each stock such that (1) the ACL does not exceed the ABC and (2) the ACT (if used) does not exceed the ACL. Because the $P^{*}$ described here is only one buffer of a multibuffer system, it seems reasonable to set $P^{*}$ higher than if it were the only buffer. A plausible range for $P^{*}$ might be values that are at least 0.25 but less than 0.50 . Assigning a value to $P^{*}$ is necessarily somewhat subjective because it will generally reflect risk tolerance as well as scientific considerations. Nonetheless, we think that making that decision explicit-rather than burying it as an implicit value in an ad hoc procedure-contributes to clarity and transparency in fishery management.

Once the value of $P^{*}$ has been chosen, methods based on probabilities are desirable because they are clearly defined, repeatable, and computable from standard assessment outputs (or clear assumptions about variance). They emphasize the need for explicit specification of the allowable probability $\left(P^{*}\right)$ of exceeding the $F_{\text {lim }}$, and perhaps most importantly, are easily communicated.

\section{Acknowledgments}

This work was supported by the Southeast Fisheries Science Center (U.S. Department of Commerce, National Oceanic and Atmospheric Administration [NOAA], National Marine Fisheries Service). Opinions expressed are those of the authors and do not constitute NOAA interpretations of any law, regulation, or policy. Our illustrations are not intended as stock assessments and do not supersede existing assessments. The methods described here have been discussed extensively in SSCs of several fishery management councils and in other venues; we thank our colleagues for their perspectives, which have undoubtedly improved our thinking. We are grateful to P. B. Conn, E. H. Williams, and D. S. Vaughan for reviews of an earlier version and to S. Atran, S. Cadrin, J. Carmichael, J. McGovern, and G. Waugh for more recent reviews. Remaining errors are the responsibility of the authors.

\section{References}

Caddy, J. F., and R. McGarvey. 1996. Targets or limits for management of fisheries? North American Journal of Fisheries Management 16:479-487.

Eagle, J., and B. H. Thompson Jr. 2003. Answering Lord Perry's question: dissecting regulatory overfishing. Ocean and Coastal Management 46:649-679.

Efron, B. 1987. Better bootstrap confidence intervals. Journal of the American Statistical Association 82:171-200.
Legault, C. M., J. E. Powers, and V. R. Restrepo. 2001. Mixed Monte Carlo/bootstrap approach to assessing king and Spanish mackerel in the Atlantic and Gulf of Mexico: its evolution and impact. Pages 37-44 in J. L. Berkson, L. L. Kline, and D. J. Orth, editors. Incorporating uncertainty into fishery models. American Fisheries Society, Symposium 27, Bethesda, Maryland.

Lunn, D. J., A. Thomas, N. Best, and D. Spiegelhalter. WinBUGS - a Bayesian modelling framework: concepts, structure, and extensibility. Statistics and Computing 10:325-337.

Methot, R. D. 1989. Synthesis model: an adaptive framework for analysis of diverse stock assessment data. International North Pacific Fisheries Commission Bulletin 50:259-277.

MSRA (Magnuson-Stevens Fishery Conservation and Management Reauthorization Act of 2006). 2006. U.S. Public Law 109-479, 120 Statute 3575.

Pella, J. J. 1967. A study of methods to estimate the Schaefer model parameters with special reference to the yellowfin tuna fishery in the eastern tropical Pacific Ocean. Doctoral dissertation. University of Washington, Seattle.

Prager, M. H. 1994. A suite of extensions to a nonequilibrium surplus-production model. Fishery Bulletin 92:374-389.

Prager, M. H. 1995. User's manual for ASPIC: a Stock Production Model Incorporating Covariates, program version 3.6x, 4th edition. National Marine Fisheries Service, Southeast Fisheries Science Center, Miami Laboratory Document MIA-2/93-55, Miami.

Prager, M. H., and A. D. MacCall. 1988. Sensitivities and variances of virtual population analysis as applied to the mackerel, Scomber japonicus. Canadian Journal of Fisheries and Aquatic Sciences 45:539-547.

Prager, M. H., C. E. Porch, K. W. Shertzer, and J. F. Caddy. 2003. Targets and limits for management of fisheries: a simple probability-based approach. North American Journal of Fisheries Management 23:349-361.

R Development Core Team. 2008. R: a language and environment for statistical computing. R Foundation for Statistical Computing, Vienna. Available: www. R-project.org. (October 2008).

Schaefer, M. B. 1957. A study of the dynamics of the fishery for yellowfin tuna in the eastern tropical Pacific Ocean. Bulletin of the Inter-American Tropical Tuna Commission 2:247-268.

Seber, G. A. F. 1973. The estimation of animal abundance and related parameters. Hafner Press, New York.

SEDAR (Southeast Data Assessment and Review). 2004. SEDAR 4 Stock assessment report 1: stock assessment of the deepwater snapper-grouper complex in the south Atlantic. South Atlantic Fishery Management Council, North Charleston, South Carolina.

Shertzer, K. W., M. H. Prager, and E. H. Williams. 2008. A probability-based approach to setting annual catch levels. Fishery Bulletin 106:225-232.

U.S. Office of the Federal Register 2009. Magnuson-Stevens Act provisions: national standard 1-optimum yield. Federal Register 74:11(16 January 2009):3178-3213. Available: ecfr.gpoaccess.gov/ (March 2009).

Venables, W. N., and B. D. Ripley. 2002. Modern applied statistics with S, 4th edition. Springer, New York. 